\title{
Skin Deep: Enhanced Variable May Help Explain Racial Disparities in Type 2 Diabetes and Prediabetes
}

\author{
Celia C. Lo · Joanna Lara · Tyrone C. Cheng
}

Received: May 12, 2017 / Published online: June 14, 2017

(C) The Author(s) 2017. This article is an open access publication

\section{ABSTRACT}

Introduction: The study refined definitions of type 2 diabetes and prediabetes (Pre-/T2D) via its four-category outcome variable. Respondents were identified as Pre-/T2D on the basis of (a) doctor's diagnosis only (i.e., managed Pre-/ T2D); (b) biomarker only (i.e., undiagnosed Pre-/T2D); or (c) both diagnosis and biomarker (i.e., unmanaged Pre-/T2D). The reference was Pre-/T2D not indicated. We linked the outcome to social structural and social support factors, health care-related factors, mental disorder, and lifestyle variables, for each racial/ethnic group. Methods: We used the 2011-2012 and 2013-2014 National Health and Nutrition Examination Surveys to measure the four-category outcome and examine race/ethnicity's role in explaining the outcome.

Enhanced content To view enhanced content for this article go to http://www.medengine.com/Redeem/ 8B98F0604AB3F244.

C. C. Lo (ه)

Department of Sociology and Social Work, Texas

Woman's University, Denton, TX, USA

e-mail: clo@twu.edu

J. Lara

Department of Sociology, Indiana University,

Bloomington, IN, USA

T. C. Cheng

Department of Social Work and Child Advocacy,

Montclair State University, Montclair, NJ, USA
Results: We found Pre-/T2D to be associated with age, BMI, physical activity, income, education, receiving health services, and other factors. A moderating role for race/ethnicity was also confirmed.

Conclusion: The racial disparities observed in our three main categories generally resulted from high levels of undiagnosed Pre-/T2D and high levels of diagnosed but unmanaged Pre-T2D. Race/ethnicity's moderating role generally indicated that, through the factors BMI, age, and receiving health services, minority status (with its attendant disadvantages) could facilitate undiagnosed Pre-/T2D as well as Pre-/ T2D indicated concurrently by diagnosis and biomarker.

Keywords: Biomarker; Multiple disadvantage model; Racial disparities; Type 2 diabetes and prediabetes (Pre-/T2D); Undiagnosed Pre-/T2D; Unmanaged Pre-/T2D

\section{INTRODUCTION}

Type 2 diabetes is the body's inability to secrete enough insulin to overcome insulin resistance [1]. It is a chronic metabolic condition that is often asymptomatic yet requires lifelong medical care as well as self-management of insulin levels and of health. Type 2 diabetes and prediabetes (Pre-/T2D) can exist without revealing itself to the people who have it [2]. Though it 
may seem common knowledge that overweight and obesity raise the odds of developing type 2 diabetes, many people are simply not knowledgeable about health, including their own health status [3]. Moreover, knowing one has Pre-/T2D is no guarantee of the self-care (medication compliance, advisable food choices, regular physical activity) that combats Pre-/ T2D's harm [4]. Undetected risk or presence of type 2 diabetes can also result from absent access to and utilization of health care [5].

A 2012 study found that $9.3 \%$ of the USA's population had diabetes; that is about 29.1 million Americans, of whom nearly onethird (28\%) had not been diagnosed as diabetic [6]. Furthermore, research has found that 37\% of Americans aged 20 years and older have prediabetes, although just $11.1 \%$ of those adults have been informed of their condition by practitioners [7]. Between $90 \%$ and $95 \%$ of people diagnosed with diabetes have type 2 diabetes, which (unlike type 1 diabetes) is believed to stem from lifestyle factors that make the body's regulation of blood sugar ineffective [6]. These lifestyle factors are strongly related to socioeconomic status (SES) and race/ethnicity [8], and changing them could significantly cut the risk that prediabetes will develop into diabetes-also cutting, when diabetes does develop, the risk of diabetes complications [9].

Previous research has shown type 2 diabetes to be relatively likely among the socioeconomically underprivileged and among members of racial/ethnic minorities [10]. But, in the absence of access to and utilization of health care, getting a doctor's diagnosis of Pre-/T2D will not be likely $[5,11]$. In other words, where health-care access/utilization exists, the rate of doctor-diagnosed Pre-/T2D should rise. Similarly, the opportunity to change one's lifestyle for the long term-for example by becoming physically active and adopting a healthier diet-is required for improving Pre-/T2D. Since diabetes is chronic, managing it and avoiding its complications require ongoing medical care, ongoing self-management, and ongoing support $[12,13]$.

The multiple disadvantage model is appropriate for thinking about how SES is related to Pre-/T2D and about the role race/ethnicity could play in the relationship [14]. Lo et al. [14] posited that disadvantages arising from social locations and social status (notably SES and race/ethnicity), as well as debilitated social support and networks, offer a context in which to understand life expectancy. Experiencing social disadvantages is stressful and can result in emotional distress and mental health problems, leading to physical illness and death $[15,16]$. The multiple disadvantage model holds, moreover, that when good-quality health services addressing distress and mental health are not accessible, additional disadvantages depleting health and fostering mortality often ensue. The model also attributes unhealthy lifestyles (overeating, neglecting physical exercise, heavy drinking, and the like) directly to disadvantaged social conditions, low SES, and minority racial/ ethnic identity.

In addition to delineating social mechanisms linking SES to health (e.g., Pre-/T2D status), the multiple disadvantage model was also developed to explain racial/ethnic disparities in health [17]. It has been shown empirically that disadvantages tending to characterize racial/ ethnic minority groups (not the white majority) underlie these groups' long occupation of the social ladder's lower rungs. One example is the groups' isolated, under-resourced residential neighborhoods; these lack both walkable spaces and outlets for healthy foods $[18,19]$. Another example is mentally ill and/or substance-abusing minority Americans' tendency to have more-serious, more-debilitating psychological/ behavioral conditions than white Americans do $[16,20]$. Two further examples of disadvantages are attitudes and beliefs about healthful habits and health-related behavior [21, 22] and relatively poor-quality health services [16]. Any and all of these contribute substantially to SES- and race/ethnicity-based health disparities, whose existence cannot be denied [19].

The present study was intended to achieve two goals. First, we wanted to redefine Pre-/T2D by relying on both doctor's diagnosis of and a certain biomarker of the condition. Pre-/T2D as redefined for this study employs four categories: Pre-/T2D indicated by doctor's diagnosis only (i.e., managed Pre-/T2D); Pre-/T2D indicated by biomarker only (i.e., undiagnosed Pre-/T2D); Pre-/T2D indicated by both doctor's diagnosis 
and biomarker (in effect, unmanaged Pre-/T2D); and Pre-/T2D not indicated. The newly developed variable can consider these categories simultaneously, using Pre-/T2D not indicated as the reference.

Second, upon adding scientific sophistication to the Pre-/T2D measure, we wanted to better acknowledge the role of social structural factors in Pre-/T2D. To do so, we applied the multiple disadvantage model to explain a complex Pre-/T2D outcome, as well as to explain any racial differences characterizing that outcome. The literature identifies no mechanisms definitively, but Pre-/T2D has been reported to be more prevalent among minority Americans (Asian-Americans, Hispanic Americans, non-Hispanic black Americans, American Indians/Alaska Natives) than white respondents $[6,23]$. Biology does likely play some role in this differential prevalence [24], but disadvantages associated with racial/ethnic minorities also contribute. The present study sought to demonstrate the redefined Pre-/T2D variable's efficacy by using it to analyze a national representative sample (of adults) for evidence of racial/ethnic disparities in Pre-/T2D prevalence.

\section{METHODS}

\section{Design and Sample}

Employing data from the 2011-2012 and 2013-2014 National Health and Nutrition Examination Surveys (NHANES) and our new four-category measure, we linked Pre-/T2D in adults aged 18 years and older to racial/ethnic groups identified in NHANES. Our study was approved by the Texas Woman's University Institutional Review Board, which exempted the study from further review. NHANES is a biennial project of the Centers for Disease Control and Prevention (CDC). It obtains data both from interviews and physical exams and was designed to assess health and nutritional states of American adults and children. The CDC in the 1960s began collecting survey data to inform vital health statistics. In 1999, NHANES was launched, to continue surveying Americans to gauge the emerging health of the nation. Five racial/ethnic groups were evaluated in the present study of 8695 respondents: white (3791), Asian-American (1019), black (2019), Mexican-American (1019), other Hispanic-American (847). This article does not concern any new studies with human or animal subjects performed by any of the authors.

\section{Measures}

During oral glucose tolerance testing (OGTT), levels of glycosylated hemoglobin (HbA1c) and of fasting plasma glucose (FPG) and 2-h plasma glucose (PG) are indicators of glucose metabolism [25]. The HbA1c readings and doctor diagnosis self-reports that had been collected for NHANES were sufficient to measure respondents using our new four-category variable. In NHANES, an HbA1c reading of 5.7 or higher places a respondent in the category presence of type 2 diabetes or prediabetes as indicated by the biomarker; respondents with HbA1c below 5.7 are classified no Pre-/T2D indicated by biomarker [13]. Our four-category Pre-/T2D outcome included the following categories: presence of Pre-/T2D indicated by doctor's diagnosis; presence of Pre-/T2D indicated by biomarker; presence of Pre-/T2D indicated by both doctor's diagnosis and biomarker; and the reference category, no Pre-/T2D indicated.

To explain Pre-/T2D, we included in the model seven social structural and support factors, two health-care access and utilization factors, one mental illness measure, four lifestyle factors, and one control variable. For the social structural factors, we allowed respondents' education and family income to measure their SES. Education level was a continuous variable ranging from 1 (less than 9th grade) to 5 (college graduate or above); it stated the highest grade level completed by a respondent. Family income, in turn, was continuous, offered responses ranging from 1 (less than US\$5000) to 15 (US\$100,000 and over). The measure for respondent age gave the exact age in years, ranging from 18 to 80 and older. Gender was coded 1 (male) or 0 (female). We created an immigration status variable offering the responses 1 (immigrant) and 0 (US-born). The dichotomous variable own home was used to indicate whether (1) or not (0) the home residence was owned/ 
Table 1 Descriptive statistics of all included variables $(N=8695)$

\begin{tabular}{|c|c|c|c|c|c|c|c|}
\hline Variable (range of values) & $\begin{array}{l}\text { White } \\
\text { Mean or } \\
\%\end{array}$ & $\begin{array}{l}\text { Asian } \\
\text { Mean or } \\
\%\end{array}$ & $\begin{array}{l}\text { Black } \\
\text { Mean or } \\
\%\end{array}$ & $\begin{array}{l}\text { Non-Mexican } \\
\text { Hispanic } \\
\text { Mean or \% }\end{array}$ & $\begin{array}{l}\text { Mexican } \\
\text { Mean or } \\
\%\end{array}$ & $\begin{array}{l}\text { Total } \\
\text { Mean or } \\
\%\end{array}$ & $p$ value \\
\hline Pre-/T2D & & & & & & & 0.00 \\
\hline Not indicated & $64.1 \%$ & $60.4 \%$ & $47.4 \%$ & $57.4 \%$ & $56.8 \%$ & $58.3 \%$ & \\
\hline $\begin{array}{l}\text { Indicated by doctor's diagnosis } \\
\text { only }\end{array}$ & $3.9 \%$ & $2.6 \%$ & $3.5 \%$ & $3.2 \%$ & $4.8 \%$ & $3.7 \%$ & \\
\hline Indicated by $\mathrm{HbAlc}$ only & $17.9 \%$ & $23.6 \%$ & $29.5 \%$ & $23.5 \%$ & $20.8 \%$ & $22.2 \%$ & \\
\hline $\begin{array}{l}\text { Indicated by doctor's diagnosis } \\
\text { and HbAlc }\end{array}$ & $14.0 \%$ & $13.5 \%$ & $19.7 \%$ & $15.9 \%$ & $17.5 \%$ & $15.9 \%$ & \\
\hline \multicolumn{8}{|l|}{ Social structural and support } \\
\hline Age & 50.36 & 44.74 & 47.64 & 47.33 & 44.08 & 48.04 & 0.00 \\
\hline Male & $49.9 \%$ & $49.1 \%$ & $48.4 \%$ & $45.0 \%$ & $51.8 \%$ & $49.2 \%$ & 0.04 \\
\hline Immigrant & $3.9 \%$ & $82.3 \%$ & $9.8 \%$ & $70.0 \%$ & $52.8 \%$ & $26.6 \%$ & 0.00 \\
\hline Education & 3.73 & 4.07 & 3.41 & 3.07 & 2.69 & 3.51 & 0.00 \\
\hline Family income & 7.67 & 9.03 & 6.76 & 6.90 & 6.87 & 7.45 & 0.00 \\
\hline Own home & $66.7 \%$ & $60.3 \%$ & $49.7 \%$ & $46.6 \%$ & $58.1 \%$ & $59.0 \%$ & 0.00 \\
\hline Marital status & $59.1 \%$ & $65.9 \%$ & $40.4 \%$ & $55.6 \%$ & $62.5 \%$ & $55.6 \%$ & 0.00 \\
\hline \multicolumn{8}{|l|}{ Lifestyle } \\
\hline Vigorous activity & 0.75 & 0.95 & 0.79 & 0.74 & 0.66 & 0.77 & 0.00 \\
\hline Moderate activity & 1.58 & 1.82 & 1.37 & 1.34 & 1.37 & 1.51 & 0.00 \\
\hline BMI & 28.93 & 24.68 & 30.64 & 28.96 & 30.07 & 28.96 & 0.00 \\
\hline Recent tobacco use & $27.1 \%$ & $10.2 \%$ & $30.1 \%$ & $17.4 \%$ & $16.1 \%$ & $23.6 \%$ & 0.00 \\
\hline \multicolumn{8}{|c|}{ Access to and use of health care services } \\
\hline Health insurance & $84.4 \%$ & $81.9 \%$ & $78.2 \%$ & $70.7 \%$ & $58.1 \%$ & $78.3 \%$ & 0.00 \\
\hline Number of health care services & 2.57 & 1.95 & 2.32 & 2.14 & 1.87 & 2.32 & 0.00 \\
\hline \multicolumn{8}{|l|}{ Mental illness } \\
\hline $\begin{array}{l}\text { Mental health professional } \\
\text { consulted }\end{array}$ & $9.6 \%$ & $3.7 \%$ & $8.5 \%$ & $9.1 \%$ & $6.0 \%$ & $8.2 \%$ & 0.00 \\
\hline \multicolumn{8}{|l|}{ Control variables } \\
\hline NHANES 11-12 & $43.9 \%$ & $50.7 \%$ & $53.8 \%$ & $51.8 \%$ & $38.7 \%$ & $47.2 \%$ & 0.00 \\
\hline$N$ & 3791 & 1019 & 2019 & 847 & 1019 & 8695 & \\
\hline
\end{tabular}




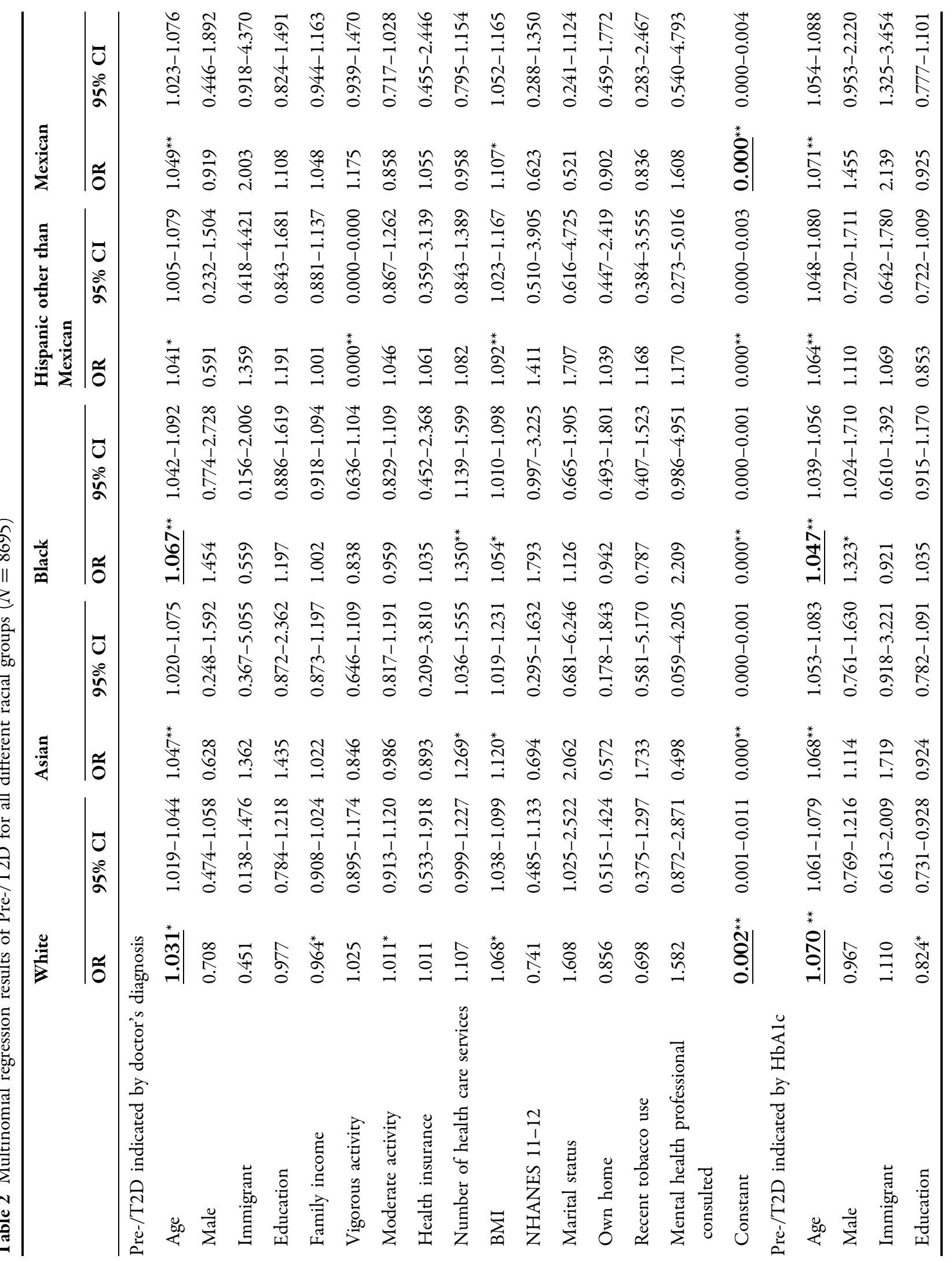




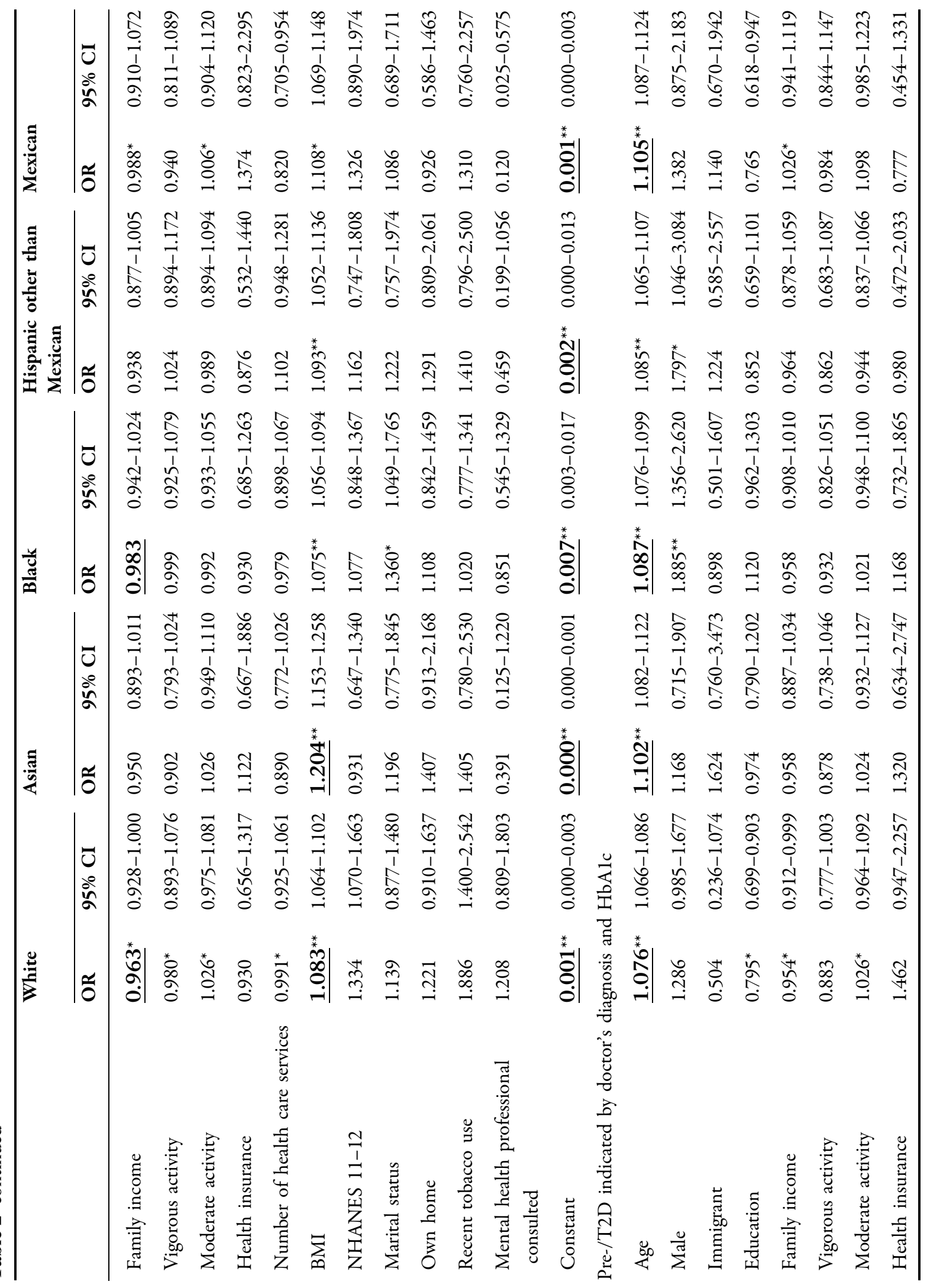




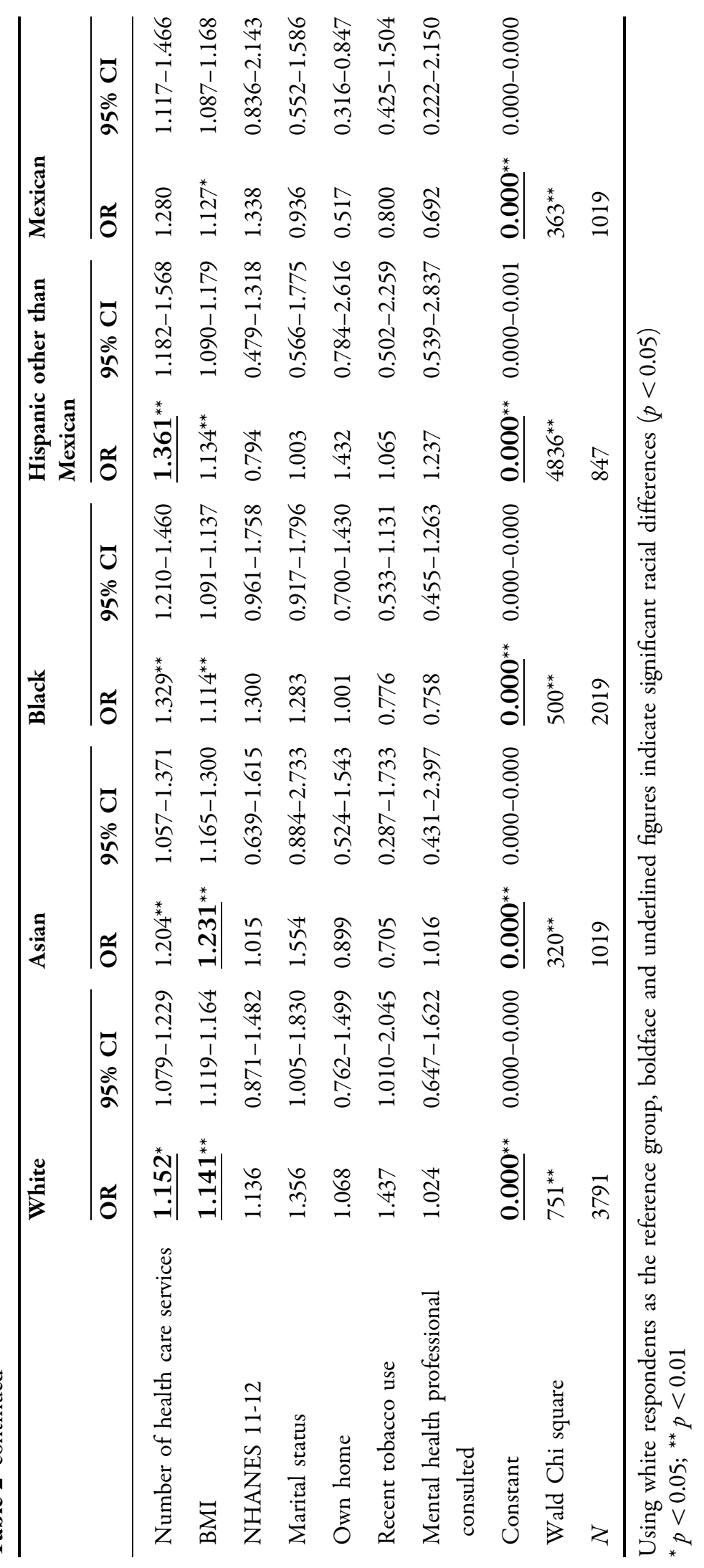


being bought. The social support variable marital status was dichotomously measured, 1 indicating those married to or living with a partner, 0 indicating otherwise.

Lifestyle factors were measured by asking respondents how many days per week they engaged in vigorous- and moderate-intensity sports/fitness/recreational activity, providing our continuous measures for vigorous activity and moderate activity. Offered responses for both variables ranged from 0 to 7 days. Each respondent's body mass index (BMI), a continuous variable and a proxy for dietary behavior, was allowed to indicate body fat. We used another dichotomous variable to describe a further lifestyle factor, current smoking status; compared to nonsmokers (0), smokers (1) are at increased risk of developing Pre-/T2D [26].

Two variables were used to indicate access to and utilization of health care. The dichotomous health insurance variable stated whether (1) or not (0) a respondent had health insurance (or a similar health care plan). In addition, the continuous variable number of times using health care in past year stated how many times during the past 12 months a respondent consulted a physician/ other health care professional at any location.

To measure mental health, we developed the dichotomous variable consulted a mental health professional, as a proxy to measure whether (1) or not (0) respondents had visited or otherwise spoken with a mental health professional (e.g., psychologist, clinical social worker, psychiatrist) during the past 12 months, about their health. To consider time within our data analyses, we created a dummy control variable of the survey year, with 1 indicating NHANES' 2011-2012 survey, 0 its 2013-2014 survey. The NHANES 2011-2012 and 2013-1014 surveys collected an adequate amount of data from individuals in the racial/ethnic groups comprised by our variable race/ethnicity: non-Hispanic white, non-Hispanic black, Mexican, Hispanic other than Mexican, and Asian.

\section{Data Analysis Strategy}

We used multinomial logistic regression techniques to examine Pre-/T2D's associations with the social structural, social support, health-care access and use, mental illness, and lifestyle factors, within each racial/ethnic group. To evaluate the moderating role of our race/ethnicity variable, we first dummy coded the race variable, creating four dummy variables: Asian-American, black, Mexican, and Hispanic other than Mexican. Next, we performed a series of multinomial regressions. Each regression included (a) the four dummy-coded race variables, (b) all the independent variables, and (c) the interaction terms including the independent variable and each of the dichotomous race/ethnicity variables. Statistically significant interactions indicated significant differences between the white majority group and minority groups, in terms of the independent variable's relationship to the outcome variable. Taking a fairly parsimonious approach, we interpreted interactions only for those independent variables that had proved statistically significant $(p<0.05)$ for at least two of the five racial/ethnic groups. To facilitate the unbiased estimation of regression results, we used the sample weights from the 2011-2012 and 2013-2014 NHANES, along with STATA software.

\section{RESULTS}

We examined 8695 respondents from the two NHANES waves. Descriptive statistics are shown in Table 1. Statistically significant differences were found from one racial/ethnic group to another, concerning all included variables. Of the whole sample, $15.9 \%$ were assigned to the category Pre-/T2D indicated by both doctor's diagnosis and biomarker (i.e., HbA1c of at least 5.7); $3.7 \%$ to Pre-/T2D indicated by doctor's diagnosis only; and $22.2 \%$ to Pre-/T2D indicated by biomarker only. The group of white respondents showed the greatest likelihood of assignment to the Pre-/T2D not indicated category, $64.1 \%$ of them being thus classified [followed by Asian-Americans (60.4\%), other Hispanic-Americans (57.4\%), Mexican-Americans (56.8\%), and black respondents (47.4\%)].

Table 2 presents the multinomial regression results for Pre-/T2D for different racial/ethnic groups. In the white category, diagnosed Pre-/ 
T2D was associated with respondents who were older, who engaged in higher levels of moderate activity, who had relatively low family incomes, and who had relatively higher BMIs. White respondents with undiagnosed Pre-/T2D were older, less educated, of relatively low family income, engaged in lower levels of vigorous activity, engaged in higher levels of moderate activity, with relatively higher BMIs, and with relatively fewer health care services in the past year. White respondents shown to have Pre-/T2D by both doctor's diagnosis and HbA1c were older, less educated, of relatively low family income, engaged in higher levels of moderate activity, with relatively many health care services used in the past year, and with relatively high BMIs.

For our Asian-American and black respondents alike, being older and having relatively high BMI were associated with higher odds for the categories diagnosed Pre-/T2D, undiagnosed Pre-/T2D, and presence of Pre-/T2D by doctor's diagnosis and HbA1c. A greater number of health care services in the past year also was found to be associated significantly with diagnosed Pre-/T2D and having Pre-/T2D indicated by doctor's diagnosis and HbA1c. Among the black respondents, being married or living with a partner increased the likelihood of having undiagnosed Pre-/T2D.

For both Mexican and non-Mexican Hispanic respondents, being older and having relatively high BMI were associated with diagnosed Pre-/T2D, undiagnosed Pre-/T2D, and presence of Pre-/T2D indicated by doctor's diagnosis and HbA1c. For Mexican-American respondents, having undiagnosed Pre-/T2D was more likely when family income was relatively low and level of moderate activity was relatively high. For this racial/ethnic group as well, diagnosed Pre-/T2D indicated by HbA1c was associated with relatively high family income. For non-Mexican Hispanic respondents, less engagement in vigorous activity increased the odds of diagnosed Pre-/T2D; male gender and greater numbers of health care services in the past year were associated with increased likelihood of Pre-/T2D's presence indicated by concurrent physician diagnosis and HbA1c.

With the procedures described in the data analysis strategy section, we evaluated race/ ethnicity's role in Pre-/T2D's associations with the specified factors. We observed 18 interactions to be statistically significant. Compared to white respondents, black respondents who were older were relatively likely to have Pre-/T2D diagnosed by a doctor. After all independent variables in the model were controlled, diagnosis of Pre-/T2D was more likely for white and for Mexican-American respondents. The likelihood of having undiagnosed Pre-/T2D was higher for younger black respondents than for younger whites. The observed inverse relationship of family income and likelihood of undiagnosed Pre-/T2D was stronger for white respondents than for black respondents. Higher BMI increased the likelihood of undiagnosed Pre-/T2D among Asian-Americans more than it did among whites. After all independent variables had been controlled, the likelihood of undiagnosed Pre-/T2D was highest among black respondents, followed by non-Mexican Hispanics, whites, Mexican-Americans, and Asian-Americans.

Compared to older white respondents, older minority respondents in all groups but non-Mexican Hispanics were relatively likely to have Pre-/T2D indicated by doctor's diagnosis and HbA1c. Greater numbers of health care services in the past year were associated with relatively high likelihood of Pre-/T2D indicated by doctor's diagnosis and HbA1c; the association was stronger for non-Mexican Hispanics than it was for white respondents. The likelihood of having Pre-/T2D indicated concurrently by doctor's diagnosis and HbA1c rose when BMI was relatively high, a relationship that was stronger among Asian-Americans than white respondents. After all independent variables had been controlled, we observed that the likelihood of having this dual doctor's diagnosis/HbA1c indication of Pre-/T2D was highest for white respondents, followed by Non-Mexican Hispanic, black, Mexican-American, and then Asian-American respondents.

\section{DISCUSSION}

Two major goals characterized our study. The first was bringing Pre-/T2D's social aspect, in 
addition to its medical aspect, to bear in a redefined Pre-/T2D [27] variable. This was achieved by creating a new four-category variable to measure the condition via three indicators: doctor's diagnosis, biomarker (HbA1c), and the two together. The second goal of our study was to link the variously categorized forms of Pre-/T2D to race/ethnicity. Identification of such links would allow us to seek social mechanisms explaining racial disparities between any of the three main categories in the new variable, and its reference category, Pre-/T2D not indicated. In performing multinomial regression modeling for each racial/ethnic group and comparing results, we were able to specify variables with links to the type of Pre-/T2D indicator noted (diagnosis, biomarker, both). We also identified variables responsible for racial differences in type of Pre-/T2D indicator noted. Four important interpretations and implications merit elaboration.

First, we observed the four-category Pre-/T2D variable to reduce selection bias typically associated with the common "presence/absence" dichotomous measure of type 2 diabetes; this should lead to improved understanding of racial disparities in health. Doctor's diagnosis is typically deemed definitive confirmation of a health problem's presence. Yet not all people who have type 2 diabetes have seen a doctor for diagnosing [6]. Even seeing a doctor may not mean being diagnosed, since the consultation may have nothing to do with diabetes symptoms. As an indicator of Pre-/T2D, doctor's diagnosis is vulnerable to potent social structural factors, including access to and utilization of health care, trust in the medical system, and resources available to pay health-care fees [28]. Moreover, for individuals diagnosed with Pre-/T2D, social structural factors also influence the efficacy of self-care [4]. Explaining more fully the burgeoning Pre-/T2D phenomenon will require us to attend equally to the condition's medical and social aspects, which are captured in our new categories for those diagnosed with Pre-/T2D and managing the disease; those undiagnosed with Pre-T2D yet exhibiting biomarkers of the disease; and those diagnosed and failing to manage the disease well. Extant research has observed the significance of undiagnosed Pre-/
T2D and its underlying reflection of public health matters [3], but rarely if ever in the literature has simultaneous analysis of three discrete Pre-/T2D categories been reported.

Second, when we applied the multiple disadvantage model, we gained empirical support for the power of some social structural and social support factors, health care-related factors, lifestyle variables, and a mental disorder measure to explain the three Pre-/T2D categories (as compared with the reference group). In general we observed that, across racial/ethnic groups, social structural factors including age, education, and family income helped predict likelihood of Pre-/T2D. As expected, advancing years and limited schooling and income were linked to greater likelihood of the three main categories of Pre-/T2D. Across these main categories, relatively higher BMI and relatively more activity of a moderate nature were uniformly associated with greater Pre-/T2D likelihood. As for the number of health care services received, its role was more complex; different directions were observed for it depending on which of the main categories was considered. Greater numbers of received health care services increased likelihood both of diagnosed Pre-/T2D and of diagnosed and "biomarked" (HbA1c) Pre-/T2D. This finding points up, first, how services may improve diagnosis of Pre-/T2D; but also, second, how unmanaged disease may prompt repeated seeking of services as symptoms persist or worsen. We observed here, however, a negative relationship between services received and biomarker-indicated Pre-/T2D, implying health care's importance for identifying previously undiagnosed disease.

Our independent variables' (social structural and social support factors, health care-related factors, lifestyle variables, mental health measure) power to explain Pre-/T2D was confirmed via observation of the intercept values from each of the models, for each racial/ethnic group. In the bivariate analysis, we found black respondents to be more likely than white respondents to have Pre-/T2D indicated by HbA1c only or by both doctor's diagnosis and HbA1c; and Mexican-Americans to be more likely than white respondents to have Pre-/T2D indicated by doctor's diagnosis. In the 
multivariate analysis, after controlling for all independent variables, we found that white respondents likelihood of Pre-/T2D indicated by doctor's diagnosis was higher than black respondents'; white respondents in the study had the highest likelihood of Pre-/T2D indicated by both diagnosis and biomarker.

Third, since we observed a significant moderating role for race/ethnicity, it is implied that the included factors (social structural and social support, health care-related, mental illness, lifestyle) are differentially associated with Pre-/T2D and account at least partially for racial disparities in prediabetes and type 2 diabetes. According to our results, compared to white respondents, black respondents were older when Pre-/T2D was identified via diagnosis or via both diagnosis and HbA1c; but black respondents were younger when Pre-/T2D was identified via HbA1c only. Compared to white respondents, our minority respondents were socially disadvantaged in three ways: (a) While rising family income decreased whites' likelihood of Pre-/T2D significantly, the association was not observed for black respondents; (b) While rising BMI levels increased the likelihood of biomarker-indicated Pre-/T2D and of Pre-/T2D indicator by diagnoses and biomarker alike among both Asian-Americans and white respondents, the association appears stronger for Asian-Americans than for whites; and (c) increased number of health care services was associated with higher likelihood of Pre-/ T2D identified via both diagnosis and HbA1c, the association was significantly stronger for non-Mexican Hispanics than for white respondents.

Finally, we must briefly note how our findings explaining Pre-/T2D cast serious doubt on the common view that type 2 diabetes starts with overeating and a sedentary lifestyle and can be stopped simply by eating less and exercising more. The racial disparities our study identified in the three main categories of its outcome variable often resulted from high levels of undiagnosed Pre-/T2D and of diagnosed but unmanaged Pre-T2D. Knowing that respondents in four minority groups were more likely than white respondents to be assigned to biomarker-only indication of Pre-/T2D and to dual indication of Pre-/T2D (biomarker and doctor's diagnosis) may hold the key to new, more effective antidiabetes tactics, we believetactics that might diminish racial health disparities. In our study, minority groups proved as or more likely than whites to have Pre-/T2D indicated only by diagnosis (see Table 1 ). The general lack of racial differences observed for this category is encouraging, suggesting people of all backgrounds can manage Pre-/T2D and minimize its complications. Of respondents in categories other than the reference, however, only $3.7 \%$ were managing their Pre-/T2D. It appears, then, that racial disparities in Pre-/T2D actually stem from undiagnosed Pre-/T2D and from poor self-care following diagnosis. Thus it could prove helpful for community health centers and other organizations to spread the word about BMI's stronger effects on Pre-/T2D among Asian Americans versus the majority; and about the comparatively early development, but late diagnosis, of Pre-/T2D among minorities versus white respondents.

Three limitations on our study deserve mention. First, the NHANES data set provided secondary cross-sectional data, the use of which precluded confirmation of cause-effect relationships between some independent variables and Pre-/T2D. Overall, however, because NHANES offers data on biomarker indication and doctor's diagnosis alike, as well as offering many variables relevant to possible links between race/ethnicity and Pre-/T2D, the data set remains a reasonable choice for exploring a new diabetes variable.

Second, empirical links have been found between Pre-/T2D and both individual-level and contextual-level SES variables. However, individual-level data from NHANES do not describe contextual factors well enough to say much about race/ethnicity's relationship to Pre-/T2D. Our study strove to understand the relationships of individual-level social structural and social support factors, health care-related factors, lifestyle variables, mental illness, and race/ ethnicity to Pre-/T2D. It leaves to future research the incorporation of contextual-level factors in analyses employing the new four-category variable.

Third, because this study's intent was to link social structural factors to a redefined 
four-category Pre-/T2D variable, such clinical predictors as weight gain and history of hypertension were not included in the model. Our results, therefore, do not take into account racial/ethnic differences in clinical predictors, even though these may lead to racial/ethnic differences in Pre-/T2D.

\section{CONCLUSIONS}

Adding scientific sophistication to the Pre-/T2D measure, in the present study we developed a new four-category variable that improves on "presence/absence" [29] by capturing some social aspects of Pre-/T2D along with the biological. The new variable involves both doctor's diagnosis of Pre-/T2D and evaluation of a Pre-/ T2D biomarker [1]. The new variable assigns respondents to one of its four categories, allowing us to capture social factors that underlie-are ultimately responsible for-racial health disparities observed in all categories but the reference.

In measuring the Pre-/T2D variable as it did, an approach that reflects both differential access to/utilization of health care for diabetes and lifestyle factors relevant to the disease, the present study linked the variable to different racial/ ethnic groups. Race/ethnicity's moderating role in Pre-/T2D means that high BMI is more detrimental to Asian Americans than to white individuals and that higher income does not translate to better health for black individuals than it does for whites [30]. Thus, for example, public health might be facilitated by ensuring better health care access/utilization for black individuals and bringing a strong message to Asian-Americans promoting low BMI.

\section{ACKNOWLEDGEMENTS}

This article was published with support from Texas Woman's University Libraries' Open Access Fund, which has funded the article processing charges. No specific funding was received for conduct of this study. All authors made significant contributions to study design, analysis, and interpretation of data, as well as to preparing and reviewing the manuscript. All named authors meet the International Committee of Medical Journal Editors (ICMJE) criteria for authorship for this manuscript, take responsibility for the integrity of the work as a whole, and have given final approval for the version to be published. Previous presentation: some of the findings from this study were presented at the 111th American Sociological Association Annual Meeting in Seattle, USA, on August 23, 2016.

Disclosures. Celia Lo, Joanna Lara, and Tyrone Cheng have nothing to disclose.

Compliance with Ethical Guidelines. The study used data that had been collected previously, and the Texas Woman's University Institutional Review Board approved the current study and exempted it from further review. This article does not concern any new studies with human or animal subjects performed by any of the authors.

Data Availability. The 2011-2012 and 2013-2014 National Health and Nutrition Examination Surveys (NHANES) data used in the present study are available online (https:// wwwn.cdc.gov/nchs/nhanes/Default.aspx).

Open Access. This article is distributed under the terms of the Creative Commons Attribution-NonCommercial 4.0 International License (http://creativecommons.org/licenses/ by-nc/4.0/), which permits any noncommercial use, distribution, and reproduction in any medium, provided you give appropriate credit to the original author(s) and the source, provide a link to the Creative Commons license, and indicate if changes were made.

\section{REFERENCES}

1. World Health Organization. Diabetes. http://www. who.int/topics/diabetes_mellitus/en/. Accessed 1 Jan 2017.

2. Barcellos SH, Goldman DP, Smith JP. Undiagnosed disease, especially diabetes, casts doubt on some of 
reported health 'advantage' of recent Mexican immigrants. Health Aff. 2012;31(12):2727-37.

3. Franzen J, Mantwill S, Rapold R, Schulz PJ. The relationship between functional health literacy and the use of the health system by diabetics in Switzerland. Eur J Pub Health. 2014;24(6):997-1003.

4. Henderson J, Wilson C, Roberts L, Munt R, Crotty M. Social barriers to type 2 diabetes self-management: the role of capital. Nurs Inq. 2014;21(4):336-45.

5. Zhang XP, Geiss LS, Cheng YJ, Beckles GL, Gregg EW, Kahn HS. The missed patient with diabeteshow access to health care affects the detection of diabetes. Diabetes Care. 2008;31(9):1748-53.

6. Division of Diabetes Translation: National Diabetes Statistics Report, 2014. In. Atlanta: National Center for Chronic Disease Prevention and Health Promotion, CDC; 2014.

7. American Diabetes Association. Fast facts: data and statistics about diabetes. Arlington: American Diabetes Association; 2014.

8. Cockerham WC. New directions in health lifestyle research. Int J Public Health. 2007;52(6):327-8.

9. Meigs JB. Multiple biomarker prediction of type 2 diabetes. Diabetes Care. 2009;32(7):1346-8.

10. Piccolo RS, Duncan DT, Pearce N, McKinlay JB. The role of neighborhood characteristics in racial/ethnic disparities in type 2 diabetes: results from the Boston Area Community Health (BACH) Survey. Soc Sci Med. 2015;130:79-90.

11. Denham SA, Wood LE, Remsberg K. Diabetes care: provider disparities in the US Appalachian region. Rural Remote Health. 2010;10(2):1320.

12. American Diabetes Association. Standards of medical care in diabetes-2011. Diabetes Care. 2011;34:S11-61.

13. Lyons TJ, Basu A. Biomarkers in diabetes: hemoglobin A1c, vascular and tissue markers. Transl Res. 2012;159(4):303-12.

14. Lo CC, Howell RJ, Cheng TC. Explaining blackwhite differences in homicide victimization. Aggress Violent Behav. 2013;18(1):125-34.

15. Lo CC, Cheng TC. Discrimination's role in minority groups' rates of substance-use disorder. Am J Addict. 2012;21(2):150-6.

16. Lo CC, Cheng TC, Howell RJ. Access to and utilization of health services as pathway to racial disparities in serious mental illness. Community Ment Health J. 2014;50(3):251-7.

17. Lo CC, Howell RJ, Cheng TC. Disparities in whites' versus blacks' self-rated health: social status, health-care services, and health behaviors. J Community Health. 2013;38(4):727-33.

18. Smalls BL, Gregory CM, Zoller JS, Egede LE. Effect of neighborhood factors on diabetes self-care behaviors in adults with type 2 diabetes. Diabetes Res Clin Pract. 2014;106(3):435-42.

19. Gaskin DJ, Thorpe RJ Jr, McGinty EE, et al. Disparities in diabetes: the nexus of race, poverty, and place. Am J Public Health. 2014;104(11):2147-55.

20. Kip KE, Peters RH, Morrison-Rodriguez B. Commentary on why national epidemiological estimates of substance abuse by race should not be used to estimate prevalence and need for substance abuse services at community and local levels. Am J Drug Alcohol Abuse. 2002;28(3):545-56.

21. Hu J, Amirehsani K, Wallace DC, Letvak S. Perceptions of barriers in managing diabetes perspectives of hispanic immigrant patients and family members. Diabetes Educ. 2013;39(4):494-503.

22. Jones RA, Utz SW, Williams IC, et al. Family interactions among African Americans diagnosed with type 2 diabetes. Diabetes Educ. 2008;34(2):318-26.

23. Agency for Healthcare Research and Quality. Fact sheet: diabetes disparities among racial and ethnic minorities. Rockvilee: US Department of Health and Human Services, Public Health Services; 2001.

24. Davidson MB, Schriger DL. Effect of age and race/ ethnicity on HbA1c levels in people without known diabetes mellitus: implications for the diagnosis of diabetes. Diabetes Res Clin Pract. 2010;87(3):415-21.

25. National Research Council. Cells and surveys: should biological measures be included in social science research? In: Finch CE, Vaupel JW, Kinsella $\mathrm{K}$, editors. Commission on Behavioral and Social Sciences and Education. Washington, DC: National Academy Press; 2000.

26. Yue X, Hu H, Koetsier M, Graaff R, Han C. Reference values for the Chinese population of skin autofluorescence as a marker of advanced glycation end products accumulated in tissue. Diabet Med. 2011;28(7):818-23.

27. Ross NA, Gilmour HL, Dasgupta K. 14-year diabetes incidence: the role of socio-economic status. Health Rep. 2010;21(3):19-28. 
28. Phelan JC, Link BG, Diez-Roux A, Kawachi I, Levin B. "Fundamental causes" of social inequalities in mortality: a test of the theory. J Health Soc Behav. 2004;45(3):265-85.

29. Shih RA, Fernandes MM, Bird CE. The application of biomarker data to the study of social determinants of health. In: Bird CE, Conrad P, Fremont
AM, editors. Handbook of medical sociology. 6th ed. Nashville: Vanderbilt University Press; 2010. p. 395-417.

30. Turner RJ, Brown TN, Hale WB. Race, socioeconomic position, and physical health: a descriptive analysis. J Health Soc Behav. 2017;58(1):23-36. 